\title{
Influence of mesoscopic structures on single molecule dynamics in thin smectic liquid crystal films
}

\author{
Benjamin Schulz, ${ }^{b}$ Daniela Täuber, ${ }^{* a}$ Jörg Schuster, $^{c}$ Thomas Baumgärtel $^{a}$ and Christian von Borczyskowski ${ }^{* a}$ \\ Received 11th March 2011, Accepted 1st June 2011 \\ DOI: $10.1039 / \mathrm{c} 1 \mathrm{sm} 05434 \mathrm{a}$
}

\begin{abstract}
Mesoscopic structures in liquids have an impact on the diffusion dynamics of the constituting molecules. Smectic 8 CB liquid crystals on silicon wafers show the formation of mesoscopic structures on the $\mu \mathrm{m}$ scale at a film thickness of $200 \mathrm{~nm}$. Depending on the kind of substrate (thermally grown or native $\mathrm{SiO}_{x}$ ), we observed the formation of focal conic domains (FCDs) and a new type of terraced holes, respectively. Dynamics are described via single perylene diimide tracer molecule tracking of translational diffusion and in the case of FCDs by a combination of translation and rotation detected via fluorescence correlation spectroscopy. Tailoring perylene diimide molecules such that the optical transition dipole moment follows the liquid crystal director allows mapping out FCDs and investigating the dynamics within a single FCD.
\end{abstract}

\section{Introduction}

Liquid crystals (LCs) can be considered as a prime example for self-organized structures as their anisotropy together with the related mobility on a molecular level ensures an energy optimization in different configurations. If such systems are forced into a frustrated environment due to contrary boundary conditions, mesoscopic structure formation such as focal conic domains (FCDs), ${ }^{1}$ hemicylinders, ${ }^{2,3}$ coexistence of different film thicknesses, ${ }^{4,5}$ or instabilities close to transition temperatures ${ }^{6}$ takes place. Such frustrated systems can easily be achieved by preparing a LC film on a substrate with planar alignment conditions for the LC molecules. The strong homeotropic alignment at the LC-air interface then breaks the translational symmetry and induces frustration to the LC. While the thermodynamics of structure formation is an interesting subject in itself, the spatial mobility of LC molecules has been less often investigated and only a few related experiments have been reported (e.g. Vilfan et $\left.a l^{7}{ }^{7}\right)$. In this paper we will concentrate on the dynamics of single probe molecules following the formation of mesoscopic structures such as focal conic domains (FCDs) and a new type of terraced film (TF) in 8CB liquid crystal films on $\mathrm{Si} / \mathrm{SiO}_{x}$ interfaces. In relation to this the fundamental question arises how such systems influence the molecular mobility

${ }^{a}$ Institute of Physics and nanoMA (Center vor nanostructured Materials and Analysis), Chemnitz University of Technology, 09107 Chemnitz, Germany. E-mail: borczyskowski@physik.tu-chemnitz.de; daniela. taeuber@physik.tu-chemnitz.de; Fax: +49 (0)371 531 21819; Tel: +49 (0)37153133015

${ }^{b}$ Max Planck Institute for Dynamcis and Self-Organization, Bunsenstraße 10, 37073 Göttingen, Germany

${ }^{c}$ Frauenhofer Research Institute for Electronic Nano Systems (ENAS), Technologie-Campus 3, 09126 Chemnitz, Germany when the dimensions are reduced down to the nanoscale regime. Since these systems have potential applications as sensors in life sciences $^{8}$ or as templates for e.g. photonic crystals ${ }^{9}$ more information is needed on a microscopic scale.

As already mentioned, not much is known yet on the intrinsic molecular mobility in frustrated films. Although in particular FCDs have been known for about 100 years, ${ }^{10}$ there are no studies related to diffusion dynamics in such films. It is convenient to investigate LC dynamics via the dynamics of fluorescent tracer molecules. ${ }^{11}$ Single molecule techniques ${ }^{12}$ offer extreme detection sensitivity e.g. to NMR experiments for systems in the nanometre regime such as very thin LC films. In a recent paper ${ }^{13}$ we have reported on lateral diffusion of single dye molecule tracking in smectic $4-n$-octyl-4'-cyanobiphenyl (8CB) as a function of film thickness between 5 and $225 \mathrm{~nm}$. The orientation with respect to the LC director has been controlled via properly tailored tracer molecules. Additionally fluorescence correlation spectroscopy (FCS) revealed a heterogeneous distribution of diffusion processes, which could not be understood in terms of the known anisotropy of diffusion with respect to the LC director.

To approach this open question of diffusion dynamics in the presence of mesoscopic LC structures, we performed (smecticnematic-smectic) temperature cycling experiments in order to control the frustrated LC structure. In this paper we take the mesoscopic structures as a phenomenon in itself, which has to be investigated in the future in much more detail with respect to thermodynamic and structure characterization. However, we focus now on the impact of two different kinds of mesoscopic structures on the diffusion in LC films of about $200 \mathrm{~nm}$ thickness. This thickness is known to be just at the threshold for the formation of FCDs. ${ }^{14}$ We discuss the influence of two types of substrates (native or thermally grown $\mathrm{SiO}_{x}$ ) which turns out to be 
a critical parameter as two fundamentally different LC topographies are observed. Moreover, we will show that tailored single probe molecules are ideal tools to investigate diffusion properties with respect to the spatially resolved mesoscopic LC patterns such as FCDs.

\section{Experimental procedure}

We studied liquid crystal films of 4 - $n$-octyl-4'-cyanobiphenyl (8CB, SYNTHON Chemicals), which are in the smectic-A phase at room temperature. In order to prepare thin films, the liquid crystal (LC) was dissolved in toluene (spectroscopic grade $(>99.9 \%)$, Merck) and then spin-coated at a rotational speed of about $100 \mathrm{~Hz}$. Using a liquid crystal concentration of about 100 $\mathrm{mg} \mathrm{ml}^{-1}$ is a reliable method to prepare homogeneous films with a thickness between 200 and $250 \mathrm{~nm}$. We used silicon wafers as substrates with either native silicon oxide or $100 \mathrm{~nm}$ of thermally grown silicon oxide (Center for Microtechnologies, Chemnitz). Substrates were cleaned by piranha solution $(60 \%$ sulfuric acid, $40 \%$ hydrogen peroxide) in an ultrasonic bath at $70{ }^{\circ} \mathrm{C}$. Before the dilution of the liquid crystal, dye molecules were dissolved in toluene at very dilute concentrations (around $10^{-10} \mathrm{~mol}^{-1}$ and close to $10^{-9} \mathrm{~mol}^{-1}$ in LC). Single tracer molecules are identified by the typical blinking (fluorescence intermittency). ${ }^{13,15}$

We obtained LC films with homogeneous and flat LC-air interfaces as identified by AFM experiments. Samples were heated (annealed) up to $35^{\circ} \mathrm{C}$ (above the (bulk) smectic-nematic phase transition at $33.5^{\circ} \mathrm{C}$ ) for half an hour. At this temperature the LC films remain in the nematic phase. Further heating into the isotropic phase results in dewetting of the film with formation of droplets. For this reason we restricted ourselves to temperature cycling into the nematic phase.

As (single) tracer molecules we used two different perylene diimides (PDIs) shown in Scheme 1. One of them (o-PDI: $N, N^{\prime}-$ di-hexadecyl-perylene-3,4,9,10-tetracarboxdiimide) orients with its long molecular axis parallel to the $\mathrm{LC}$ director ${ }^{13}$ while the other one (no-PDI: $N, N^{\prime}$-di-propyl-1,6,7,12-tetra-(4-heptyl-phenoxy)-perylene-3,4,9,10-tetra-carboxdiimide) has no preferred orientation in a LC film. The orientational behavior of PDI dye

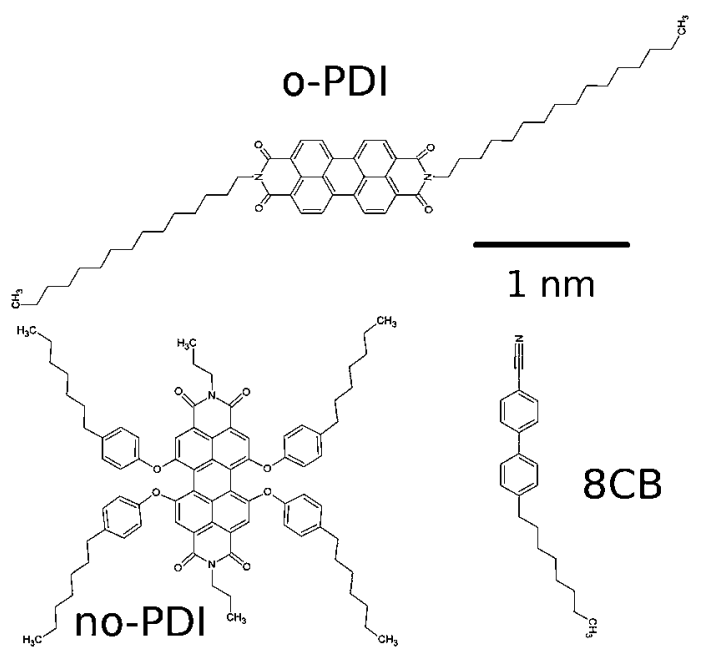

Scheme 1 Structure of tracer dye molecules (o-PDI and no-PDI) and $8 \mathrm{CB}$. molecules has been discussed recently in more detail. ${ }^{13}$ The optical transition dipole moment of PDI is parallel to the long axis of the chromophoric backbone. Therefore in the case of o-PDI the dipole moment is oriented parallel to the LC director. When the dipole moment is perfectly perpendicular to the substrate, no fluorescence emission will be detected. However, the orientation will not be perfectly parallel to the director and the order parameter of the LC will be always less than 1. This implies that considerably reduced fluorescence intensity can be observed. In fact, experimentally we always observe significantly less o-PDI molecules than no-PDI though the absolute concentration is approximately the same.

To monitor diffusion of PDI dye molecules we used a homebuilt widefield microscope. ${ }^{13}$ PDI molecules were excited at 514 $\mathrm{nm}$ (argon ion laser Innova 70C, Coherent). Separating the reflected and the fluorescent light we got a fluorescence image of the sample using an electron multiplying CCD-camera (Andor iXon 885) operating at a frame rate of $50 \mathrm{fps}$.

The analysis of the observed diffusion time traces was performed by single molecule tracking either via reconstruction of the diffusion trajectories and calculation of mean square displacements from which the diffusion coefficient for each tracked molecule (time scale $>1 \mathrm{~s}$ ) is determined, or via calculating diffusivities following the procedure described recently. ${ }^{16}$ The latter approach provides information on shorter time scales than msd analysis as this method does not average over a total trajectory but identifies the translational steps between two subsequent CCD frames. This implies a temporal resolution of $20 \mathrm{~ms}$ and a time scale between $1 \mathrm{~s}$ and $20 \mathrm{~ms}$. A much faster time resolution is obtained via fluorescence correlation spectroscopy (FCS) in a confocal setup ${ }^{13}$ resulting in a time range of $1-10^{-6} \mathrm{~s}$. For FCS measurements the dye concentration was about $10^{-8}$ mol $1^{-1}$ in toluene. AFM images have been obtained by a Nanowizard (JPK Instruments). Reflection and fluorescence images have been obtained on typical length scales of $10 \times 10 \mu \mathrm{m}$ by a laser scanning microscope (Zeiss LSM 510). Further experimental details about the setups and experimental procedures are given elsewhere. ${ }^{13}$

\section{Results}

\section{Formation of mesoscopic structures}

Directly after sample preparation we did not find any differences in structure between films prepared on silicon wafers with native oxide or those with an oxide layer of $100 \mathrm{~nm}$ thickness. However, after annealing the films into the nematic phase and slowly cooling down to the smectic phase at room temperature, two different types of mesoscopic structures emerged, depending on which kind of substrate was used.

For samples on thermally grown oxide we observe focal conic domains (LC-FCDs) that are known to form on silicon substrates ${ }^{17}$ due to the random planar anchoring condition for 8CB molecules. Scheme 2 shows schematically the structure of one FCD. The optical identification of a FCD was achieved by taking fluorescence images of a sample with a high concentration of o-PDI. These molecules align with their transition dipole parallel to the director of $8 \mathrm{CB} .{ }^{13}$ Accordingly, the probability of optical excitation as well as the probability of emission into the 


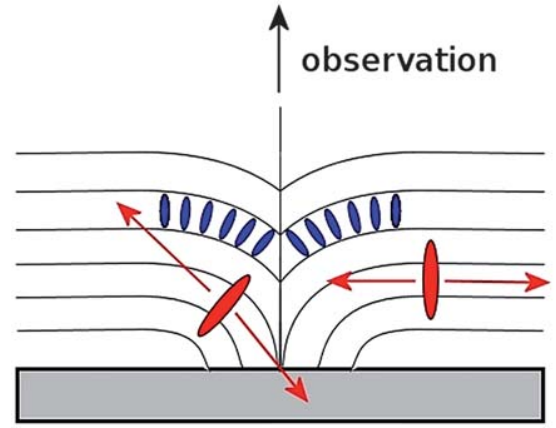

Scheme 2 Schematic presentation of a FCD with incorporated o-PDI. Preferred directions of emission of o-PDI molecules in different areas of a focal conic domain are indicated by arrows.

direction of observation is increased if the molecules are-in the center of a FCD - oriented with their long axis parallel to the substrate. Since the excitation was performed with linearly polarized laser light, both emission and reflection depend on the angle between molecular orientation and polarization of the laser. So finally for a circular FCD one obtains textures that look like two circles connected in the center of the domain. ${ }^{18}$ Exactly this kind of texture is found in our samples as shown in Fig. 1 both in reflection and fluorescence. The fluorescence image reveals the orientation of the dipole moment of the dye molecules, i.e. the brighter areas correspond to the areas where the molecules are mainly aligned along the direction of polarization of the light. On the other hand the reflection image shows the reflectivity of the surface according to the Fresnel equations, therefore providing information about the surface of the film. Thus the fluorescence image is a lot easier to interpret regarding the director orientation inside the liquid crystal. However the reflection image was also necessary as it was used to identify the structure of the samples containing dye molecule with single molecule concentration.

We obtained a size distribution of the FCD between 0.5 and $1.5 \mu \mathrm{m}$ diameter, which is slightly narrower than the one observed for thicker films. ${ }^{19}$ We did not find any regular arrangement of the domain centers.

However, for the annealed samples on native oxide we found a completely different structure (LC-TF) which is characterized
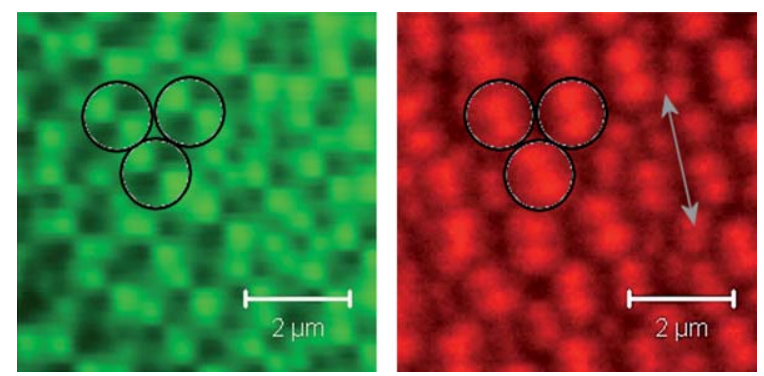

Fig. 1 Reflection (left) and PDI fluorescence (right) image of PDI doped $8 \mathrm{CB}$ on silicon with $100 \mathrm{~nm}$ oxide. Characteristic textures (see text) demonstrate the presence of focal conic domains (LC-FCDs). Open circles indicate the typical diameter of a single domain. Images were obtained from the same area of the film. The arrow marks the direction of linear polarization of incident light.
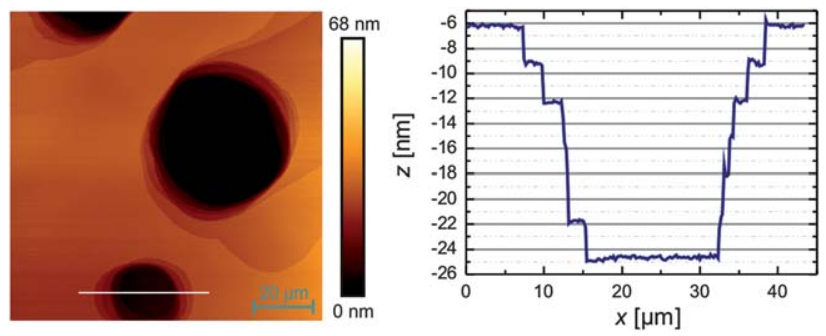

Fig. 2 Left: AFM-image of an annealed film on native oxide which shows terrace-like structures (TF). Right: cross-section along the white line of the left image which shows step sizes of multiples of about $3 \mathrm{~nm}$ which corresponds to the $3.2 \mathrm{~nm}$ height of one smectic double layer of $8 \mathrm{CB}$.

by AFM as a terraced surface surrounding deep holes. This structure remains stable for days. A typical AFM-image is shown in Fig. 2 together with a cross-section through one of the holes. While AFM only reflects height profiles the microscopic structure of the terraced holes remains open and would need detailed optical experiments similar to those reported by van Effenterre et al. ${ }^{20}$ Presently such experiments are out of scope, since we are not yet able to correlate the statistical appearance of AFM detected TF (revealing varying shape, depth and distribution) with precise optical data, which would be necessary to describe a possibly TF related birefringence. An overall measurement of birefringence is not decisive with respect to TF which reflects only a minor part of the total film volume.

To the best of our knowledge, such a structure has not been reported yet. The overall structure reminds us of the coexistence of two LC phases as described by Garcia et al. ${ }^{4}$ However, in the corresponding experiment only two defined film thicknesses were found, and the structures appeared as a new phase at the smectic-nematic transition. In contrast, we clearly see several steps. In the cross-section through one of the holes (Fig. 2, right) one can identify characteristic step heights related to the change of the film thickness. The respective height is always found to be about $3 \mathrm{~nm}$ or a multiple of it. As this corresponds to the typical thickness of smectic bilayers of $8 \mathrm{CB},{ }^{21}$ we conclude that the structure near the air-LC interface consists of parallel smectic layers without any bending of the layers as would be characteristic for a FCD. As the depth of the holes was found to be not deeper than $85 \mathrm{~nm}$, the holes are not an indication for dewetting, given the fact that we have an overall total film thickness close to $225 \mathrm{~nm}$. A similar structure has been described by Overney et $a .^{22}$ But in that case the structure was formed in the solid phase of the LC due to internal strain caused by different thermal expansion coefficients of the substrate and the (solid) LC. In our case the mobility of the molecules in the smectic phase should inhibit any strain caused by expansion. Terraced steps have also been observed in the case of wetting $8 \mathrm{CB}$ droplets on silicon wafers with native oxide using scanning polarization force microscopy. ${ }^{23}$ Therefore the strong interaction with the silicon substrate certainly plays a role.

\section{Diffusion trajectories}

To obtain information about the influence of the observed mesoscopic structures on diffusion we used single PDI molecules 
as tracer molecules for dynamic processes in LC-FCD or LC-TF. We identify trajectories related to the projection of the lateral diffusion of single molecules over at least 50 frames separated by $\Delta t$. Along those trajectories we calculate mean square displacements and make use of the two-dimensional Einstein-Smoluchowski equation

$$
D_{\mathrm{msd}}=\lim _{\Delta t \rightarrow \infty} \operatorname{msd}(\Delta t) /(4 \Delta t),
$$

to calculate diffusion coefficients $D_{\mathrm{msd}}$. By this a diffusion coefficient is ascribed to each trajectory. In Fig. 3 the obtained diffusion coefficients are shown for both types of PDI in the case of $\mathrm{LC}-\mathrm{FCD}$ and $\mathrm{LC}-\mathrm{TF}$ in comparison to the respective (LC) films before annealing. The $D_{\text {msd }}$ distributions are fitted with the assumption of only one Gaussian, which is certainly only a rough approximation. The absolute range of $D_{\mathrm{msd}}$ is in qualitative agreement with $D_{\|}$and $D_{\perp}$ for self-diffusion of $8 \mathrm{CB}$ in the smectic phase. $^{24}$ For this comparison the difference in dye diameter has been taken into account. ${ }^{13}$

Only for no-PDI we observe for both substrates a slight increase of the peak value of the distribution of the diffusion coefficient $D_{\text {msd }}$. The increase is more significant for LC-TF, where the average diffusion coefficient is increased by about $30 \%$ together with a strong broadening of the distribution (FWHM) of the coefficients. All data for $D_{\mathrm{msd}}$ are collected in Table 1.

\section{Probability distributions of diffusivities}

Since the trajectory analysis considers only traces of more than 50 sequential frames, relatively fast diffusion processes are underestimated. Such processes might show up, however, when analyzing probability distributions of single diffusivities. ${ }^{13}$ In this approach all squared displacements $r^{2}$ between all traced molecules in succeeding frames are calculated. With these data probability distributions $p\left(d_{\text {diff }}, \Delta t\right)$ of scaled squared displacements $d_{\text {diff }}=r^{2} /(4 \Delta t)$ are analyzed, ${ }^{16}$ where $\Delta t$ is the time lag between succeeding frames. Since our data are related to a projection of the three dimensional diffusion into the plane parallel to the substrate, we have to consider two-dimensional diffusion. The probability density for diffusivities can now be written as

$$
p\left(d_{\text {diff }}, \Delta t\right)=D_{\text {diff }}^{-1} \exp \left(-d_{\text {diff }} / D_{\text {diff }}\right)
$$

An elegant way to analyze experimental data is to use an integrated form of $p\left(d_{\text {diff }}, \Delta t\right)$, i.e. the complementary cumulative probability distribution $C\left(d_{\text {diff }}, \Delta t\right) .{ }^{25}$ The solutions for the twodimensional case are given by the exponential function

$$
C\left(d_{\text {diff }}, \Delta t\right)=1-P\left(d_{\text {diff }}, \Delta t\right)=\exp \left(-d_{\text {diff }} / D_{\text {diff }}\right),
$$

which yields a straight line in a semilog-plot. In the case of heterogeneous or anomalous diffusion, experimental data will deviate from this straight line. Then instead of eqn (3) a multiexponential fit to the cumulative probability distributions

$$
C\left(d_{\mathrm{diff}}, \Delta t\right)=\sum_{i} A_{i} \exp \left(-d_{\mathrm{diff}} / D_{\mathrm{diff}, i}\right) .
$$

can be used to obtain diffusion coefficients $D_{\text {diff }, i}{ }^{13,16}$ Since only no-PDI shows a noticeable dependence on structure formation, we applied the analysis only to this tracer molecule. The plots in Fig. 4 clearly show a multi-exponential behavior which is an indication for the presence of more than one diffusion process, as was already evident in Fig. 3 and Table 1 from the analysis of $D_{\text {msd }}$.

The diffusion dynamics of no-PDI in $8 \mathrm{CB}$ films on $100 \mathrm{~nm}$ $\mathrm{SiO}_{x}$ can be divided into three basically different time regimes: a slow one, which represents (partially) immobile molecules (sticking on the substrate surface $\left.{ }^{13}\right)$, a medium one $\left(A_{1}, A_{2}\right)$,
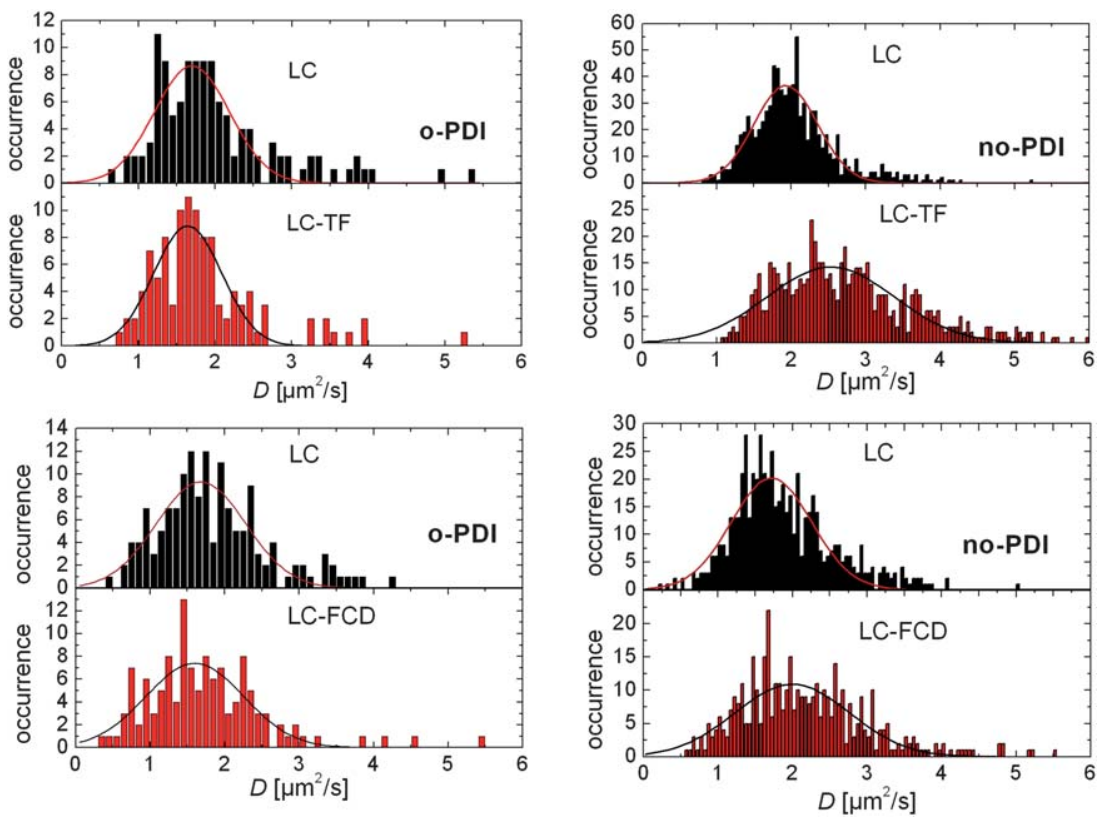

Fig. 3 Diffusion coefficients $D_{\text {msd }}$ for o-PDI (left) and no-PDI (right) in LC, LC-FCD and LC-TF films on $100 \mathrm{~nm} \mathrm{SiO} x$ (bottom) and native SiO $x$ (top). The fitted data are collected in Table 1. 
Table 1 Diffusion data from trajectory $\left(D_{\mathrm{msd}}\right)$ and diffusivity analysis $\left(D_{\mathrm{diff}}\right)^{a}$

\begin{tabular}{|c|c|c|c|c|c|c|}
\hline Dye & $\mathrm{Si} / \mathrm{SiO}_{x}$ & $8 \mathrm{CB}$ & $D_{\mathrm{msd}} / \mu \mathrm{m}^{2} \mathrm{~s}^{-1}$ & $\mathrm{FWHM} / \mu \mathrm{m}^{2} \mathrm{~s}^{-1}$ & $D_{\mathrm{diff}, 1} / \mu \mathrm{m}^{2} \mathrm{~s}^{-1}$ & $D_{\mathrm{diff}, 2} / \mu \mathrm{m}^{2} \mathrm{~s}^{-1}$ \\
\hline \multirow[t]{3}{*}{ o-PDI } & Native & $\mathrm{LC}$ & $2.6 \pm 0.3$ & $1.8 \pm 0.2$ & & \\
\hline & & LC-TF & $2.4 \pm 0.3$ & $1.5 \pm 0.2$ & & \\
\hline & & LC-FCD & $2.4 \pm 0.3$ & $2.4 \pm 0.2$ & & \\
\hline \multirow[t]{3}{*}{ no-PDI } & Native & $\mathrm{LC}$ & $2.9 \pm 0.3$ & $1.5 \pm 0.2$ & $2.9 \pm 0.2$ & \\
\hline & & LC-TF & $3.8 \pm 0.5$ & $3.0 \pm 0.3$ & & $3.4 \pm 0.2$ \\
\hline & $100 \mathrm{~nm}$ & $\mathrm{LC}$ & $2.6 \pm 0.3$ & $2.0 \pm 0.2$ & $2.4 \pm 0.3$ & $3.8 \pm 0.6$ \\
\hline
\end{tabular}

${ }^{a}$ The real diffusion coefficient in single molecule tracking experiments is about $50 \%$ higher than the apparent diffusion coefficient. ${ }^{35}$ The values in the table are therefore scaled by a factor of 1.5 compared to those depicted in Fig. 3 and 4.
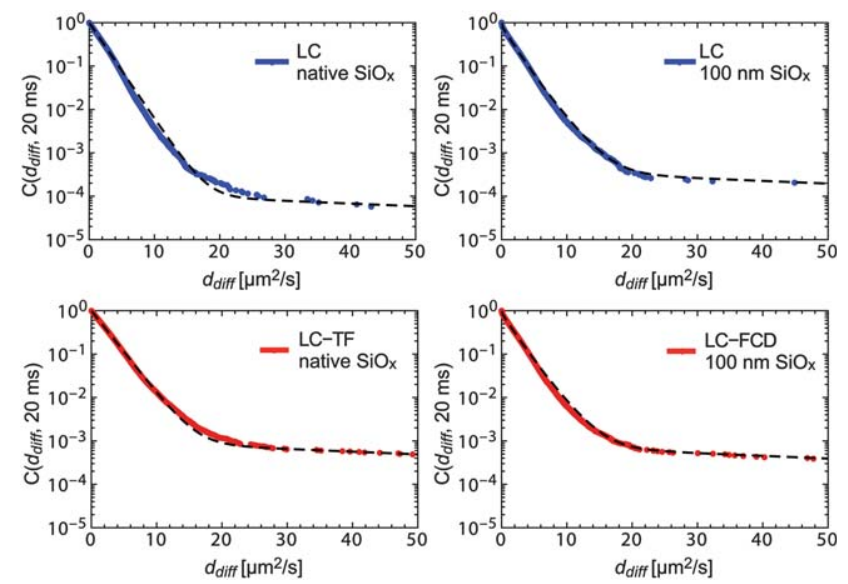

Fig. 4 Probability distributions of $d_{\text {diff }}$ (data points) on two different oxides for no-PDI, together with (broken lines) multi-exponential fits (representing diffusion on slow, medium and fast time scales) of LC (top), LC-TF (bottom left) and LC-FCD (bottom right). The fitted data are collected in Table 2.

which is in the range of the trajectory analysis, and a much faster one $\left(A_{3}\right)$ with a very small relative amplitude. Data were fitted with up to 3 diffusion coefficients. All related data are collected in Table 2. Since fluorescence is quenched close to the substrate, ${ }^{26}$ no immobile PDI is observed in the case of native oxide. In the medium time regime all $D_{\mathrm{msd}}$ and $D_{\text {diff }}$ are in rough qualitative agreement with each other. The fastest regime $\left(D_{\text {diff,3 }}>30 D_{\text {diff,2 }}\right)$ cannot be detected in the trajectory analysis but shows up as a very small contribution for all configurations (a similar fast component will show up more clearly in the fluorescence correlation spectroscopy as will be described later on). In the following we will concentrate on details in the medium time regime.
For $8 \mathrm{CB}$ films on $100 \mathrm{~nm}$ oxide we clearly observe two components $D_{\text {diff, } 1}=2.4 \mu \mathrm{m}^{2} \mathrm{~s}^{-1}$ and $D_{\text {diff, } 2}=3.8 \mu \mathrm{m}^{2} \mathrm{~s}^{-1}$, which nearly do not change in magnitude upon formation of FCD. According to diffusivity analysis $D_{\text {diff,2 }}$ is a relatively strong component, which shows up only weakly in msd analysis, probably because this type of analysis is less sensitive to relatively fast components. In that respect analysis of diffusivities is superior to msd analysis.

The situation for native oxide is distinct from the one of 100 $\mathrm{nm}$ oxide. For both cases temperature cycling leads to faster diffusion. In the case of LC-TF only one medium component $D_{\text {diff, } 2}$ can be detected, which increases from 2.9 to $3.4 \mu \mathrm{m}^{2} \mathrm{~s}^{-1}$ after annealing. Also for LC-FCD an enhancement of diffusion can be observed. Here the amplitude $A_{2}$ for $D_{\text {diff, } 2}$ increases from 0.29 to 0.37 .

\section{Fluorescence correlation spectroscopy}

The time resolution of the widefield based methods (trajectory and single step analysis) is restricted by the exposure time of the CCD camera of $20 \mathrm{~ms}$. Nevertheless, diffusivity analysis shows a small but very fast contribution, which cannot be related to the intrinsic translational (self-)diffusion typical for $8 \mathrm{CB}$, but to an additional inherent heterogeneous structure. ${ }^{13}$ In order to get a better time resolution we applied fluorescence correlation spectroscopy (FCS), which can be used to measure the dynamic behavior related to an area not larger than the size of the illuminating laser focus. Scanning the laser focus over the sample provides local information on dynamic processes below milliseconds with a lateral precision of about $20 \mathrm{~nm}$. In a previous paper ${ }^{13}$ we have argued that the relevant time scale experimentally observed by FCS is slower than predicted for molecular rotational motion. Also in the present experiments we neglect the

Table 2 Analysis of diffusivities $\left(D_{\text {diff }, i}\right.$ in $\left.\mu \mathrm{m}^{2} \mathrm{~s}^{-1}\right)$ for no-PDI ${ }^{a}$

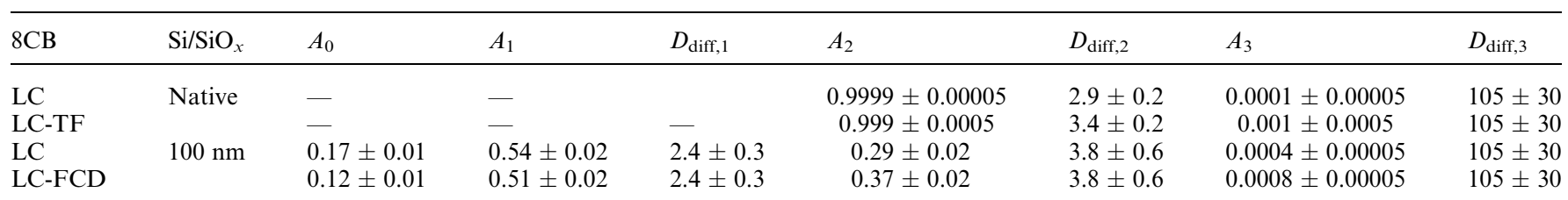

${ }^{a} A_{i}$ corresponds to the relative contribution of a multiexponential fit according to eqn (4). The real diffusion coefficient in single molecule tracking experiments is about $50 \%$ higher than the apparent diffusion coefficient. ${ }^{35}$ The values in the table are therefore scaled by a factor of 1.5 compared to those depicted in Fig. 4. 
(too fast) intrinsic molecular rotation. The FCS method makes use of the fluorescence intensity autocorrelation function

$$
G_{2}(\tau)=\langle I(t) I(t+\tau)\rangle /\langle I(t)\rangle^{2}
$$

In the case of structure formation such as LC-FCD or LC-TF one might expect a spatial dependence of the dynamics when analyzing areas with about $500 \mathrm{~nm}$ resolution (the lateral focal diameter of the exciting laser). Diffusion in the $z$-direction would not influence the correlation function as our film thickness is considerably smaller than the focal depth of the laser, which is on the order of $0.9 \mu \mathrm{m}$. In the following we first concentrate on the investigation of LC-FCD.

For simplicity let us neglect the coupled rotation in the case of o-PDI at the moment and consider the expectation for lateral diffusion only. Outside a FCD diffusion $D_{\perp}$ perpendicular to the LC director will be easily detected due to the lateral 2-D projection, while close to the center of a FCD mostly diffusion $D_{\|}$parallel to the director will be detected. Diffusion $D_{\|}$in bulk $8 \mathrm{CB}$ is roughly twice the diffusion coefficient perpendicular to the director $D_{\perp} \cdot{ }^{24}$ Furthermore, the fluorescence emission intensity of o-PDI molecules is stronger in the direction of observation when the optical dipole is oriented along the director in the center of a FCD (see Scheme 2). This leads to a higher fluorescence intensity of o-PDI in the FCD and thus we expect a higher amplitude of the correlation function in the center of a FCD. As no-PDI does not align along the LC director, a homogeneous fluorescence intensity distribution is expected for this molecule.

Due to sensitivity reasons, we were not able to determine the location of FCD in the single molecule setup directly as we succeeded according to Fig. 1. Therefore we measured the FCS signal at positions along randomly chosen lines across the sample. We assume that measurements are performed statistically in the center of a domain, between two domains or in an intermediate region.

The variations in autocorrelation functions $G_{2, \text { norm }}$ in each plot of Fig. 5 are related to different lateral positions of the laser focus on the LC-FCD sample. The respective amplitudes of the fluorescence signals have been normalized to 2 for direct comparison. It is clearly evident that the spread in decay times is significantly higher for o-PDI as compared to no-PDI. As we will discuss later in more detail, for o-PDI we find a strong variation of the amplitude $A$ of the correlation function by more than a factor of 2. However, for no-PDI no significant position dependence of amplitudes or characteristic times has been found.

Experimentally determined FCS curves cannot be fitted assuming normal diffusion according to Aragón and Pecora. ${ }^{27}$ There are several possibilities for such a deviation from the "normal" behavior as has been discussed elsewhere. ${ }^{13}$ As shown by Hac et al. $^{28}$ improvement can be obtained assuming e.g. two dynamic processes according to

$$
G_{2}(\tau)=1+A_{1} /\left(1+\tau / \tau_{D 1}\right)+A_{2} /\left(1+\tau / \tau_{D 2}\right)
$$

Despite the complex behavior of the autocorrelation function, in this publication we approximate the complex behavior while assuming anomalous dynamics and therefore fitted the data according to the following analytical approximation ${ }^{28}$

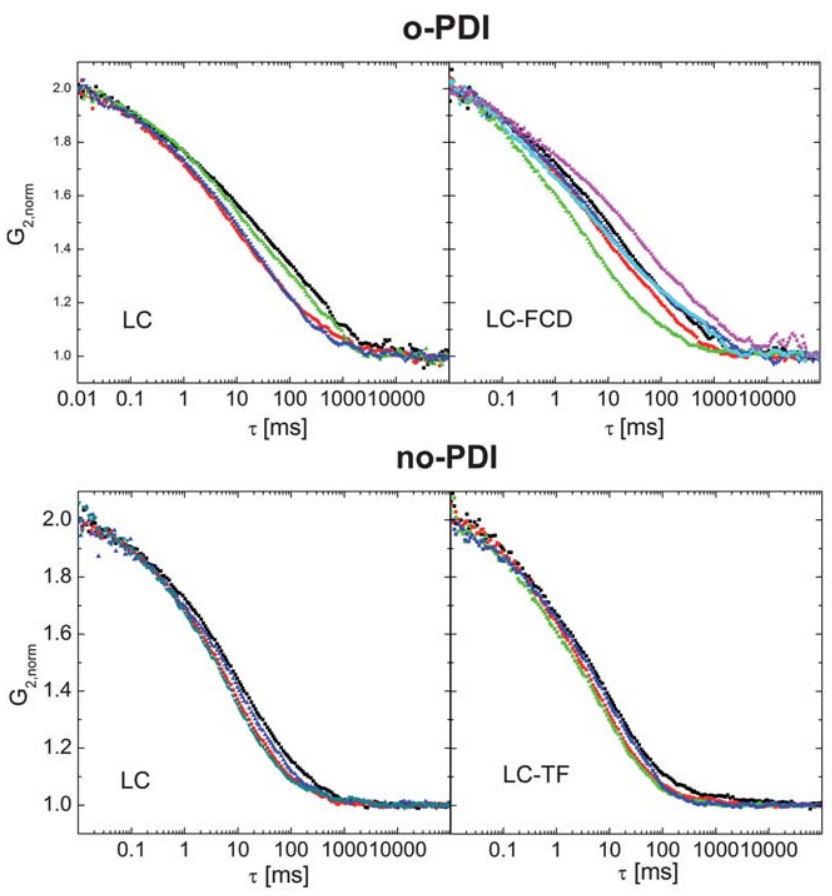

Fig. 5 Examples of normalized autocorrelation functions $G_{2, \text { norm }}$ before and after the formation of FCD for no-PDI (bottom) and o-PDI (top). We scanned the confocal spot over the sample in steps of typically $200 \mathrm{~nm}$.

$$
G_{2}(\tau)=1+A /\left[1+\left(\tau / \tau_{\mathrm{D}}\right)^{\alpha}\right]
$$

with $\alpha<1$. This approximation contains both lateral diffusion processes (medium time scale of diffusivity analysis) and much faster ones. Table 3 collects the corresponding fitting results.

First of all, there is an average decrease of about $30 \%$ for o-PDI when comparing characteristic times $\tau_{\mathrm{D}}$ for LC and LCFCD. At the same time the parameter $\alpha$ of the anomalous dynamics remains constant so that the general form of the autocorrelation function remains unchanged. Standard deviations $\sigma\left(\tau_{\mathrm{D}}\right)$ are much smaller for no-PDI as compared to o-PDI.

For a deeper insight into the correlation between the amplitude $A$ of the correlation function and the related characteristic time $\tau_{\mathrm{D}}$ for o-PDI we (arbitrarily) scanned through a focal conic domain. Since FCD diameters are in the range of about $1 \mu \mathrm{m}$, we scanned the confocal spot over the sample in steps of $200 \mathrm{~nm}$ at an arbitrarily chosen line across the sample. For a typical example the amplitude $A$ shows a clear maximum at $d=0.5-0.75$

Table 3 Analysis of data from fluorescence correlation spectroscopy

\begin{tabular}{llllll}
\hline Dye & $8 \mathrm{CB}$ & $\tau_{\mathrm{D}}{ }^{a} / \mathrm{ms}$ & $\sigma\left(\tau_{\mathrm{D}}\right)^{b} / \mathrm{ms}$ & $\alpha^{a}$ & $\sigma(\alpha)^{b}$ \\
\hline \multirow{2}{*}{ o-PDI } & LC & 3.31 & 0.60 & 0.46 & 0.04 \\
& LC-FCD & 2.22 & 0.68 & 0.50 & 0.03 \\
no-PDI & LC & 2.69 & 0.26 & 0.59 & 0.05 \\
& LC-FCD & 2.22 & 0.19 & 0.55 & 0.06
\end{tabular}

${ }^{a}$ Characteristic times $\tau_{\mathrm{D}}$ and $\alpha$ (eqn (7)) averaged over all spatial positions. ${ }^{b}$ Standard deviation. 

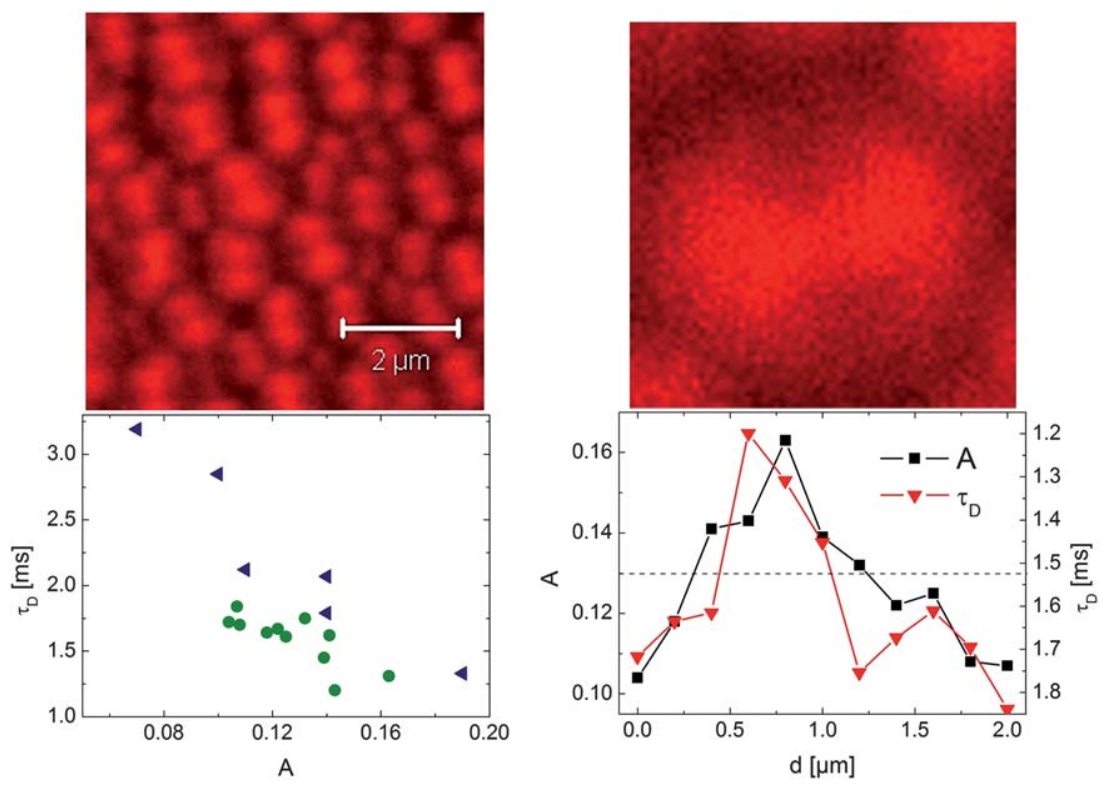

Fig. 6 Correlation between the amplitude $A$ of the autocorrelation function and the characteristic time $\tau_{\mathrm{D}}$ for o-PDI. Bottom left: $\tau_{\mathrm{D}}$ as a function of the corresponding amplitude $A$ for two independent measurements. The absolute values of $A$ depend on the arbitrarily chosen "scanning line" across a typical sample (top left). Bottom right: amplitude $A$ and $\tau_{\mathrm{D}}$ as a function of the spatial position $\mathrm{d}$ along an arbitrarily chosen scan across the sample (through a FCD) with steps of length $200 \mathrm{~nm}$. Lines are for eye guide. The broken line denotes half of the maximum intensity. On top right an image section of 2 microns size is shown for comparison. Note that the graphs on top have been selected from a different sample than the data shown below.

$\mu \mathrm{m}$ which corresponds to a minimum of $\tau_{\mathrm{D}}$ (please note the inverse scale) (Fig. 6, bottom right). The spatial widths of both curves are close to $1 \mu \mathrm{m}$ as expected for a FCD shown in Fig. 6 (top). The amplitude $A$ is related to the absolute fluorescence intensity, for which a maximum should be obtained for parallel orientation of o-PDI with respect to the surface. This finding is expected at the center of a FCD in the case of o-PDI since that dye follows the orientation of the LC director (see Scheme 2). The experimental data nicely show that they reflect the fluorescence intensity distribution within a FCD shown in the upper part of Fig. 6. Note, however, that FCS data and FCD imaging have been taken from different samples due to experimental reasons.

As shown in Fig. 6 (left) we find that $\tau_{\mathrm{D}}$ is decreasing with increasing amplitude $A$. The increase of $A$ is in agreement with the expectation that in the center of a FCD the fluorescence intensity is higher (see Scheme 2). It can also be seen in Fig. 6 (left), that the absolute range of $\tau_{\mathrm{D}}$ and $A$ is varying for different measurements. The reason for this is that (as mentioned earlier) we were not able to select defined areas of the FCD so that a scan through the center of a domain would result in a larger difference in the values for $\tau_{\mathrm{D}}$ and $A$ (blue triangles) than a scan along boundary areas of the FCD. This leads to a smaller spread of absolute values (green circles). But also in this case the relation between amplitude and correlation time is quite obvious.

For both PDI molecules the averaged $\tau_{\mathrm{D}}$ tentatively decreases (see Table 3) for LC-FCD as compared to the non-tempered LC films, which points towards overall faster dynamics. Effects are much smaller for no-PDI, since no-PDI does not follow the orientation of $8 \mathrm{CB}$ molecules.

We have also performed FCS experiments on LC-TF, which, however, did not show any difference in characteristic times $\tau_{\mathrm{D}}$ when comparing with LC data.

\section{Discussion}

While the formation and structure of FCDs are well documented, ${ }^{14,17}$ the formation of TFs has not yet been reported. The appearance of a TF is not surprising since it shows up for a thickness close to the known threshold thickness associated with FCD formation. ${ }^{14}$ It most likely reveals a change of anchoring when comparing the two substrates, due to the underlying silicon. It demonstrates that planar anchoring on native oxide is weaker. In order to assess the critical thickness at which TFs appear, films of varied thickness might be studied. However, since the focus of our experiments is on the implementation of single molecule detection schemes into the field of LC structure formation, thinner films on native oxide escape the detection due to strong fluorescence quenching caused by the silicon substrate. Additionally, thicker films would escape the detection due to the increase of optical background signal intensity.

Nevertheless, more detailed experiments are needed to clarify the exact conditions of TF formation which is beyond the scope of the present experiments, which concentrate on the influence of mesoscopic structures on diffusion dynamics. However, at the end of the discussion we will draw some conclusions related to the TF structure based on merely diffusion dynamics. Such suggestions are naturally limited and need further proof by more detailed future experiments. In Scheme 3 we suggest a possible structure of a TF based on related reports in the literature andas will be discussed later-our proposals deduced from diffusion experiments only. The suggested structure shown in Scheme 3 consists of a planar alignment of $8 \mathrm{CB}$ on the substrate, then a (newly formed) intermediate nematic layer that turns the director orientation via bend deformation from a parallel orientation of the nematic layer directly at the interface to the 


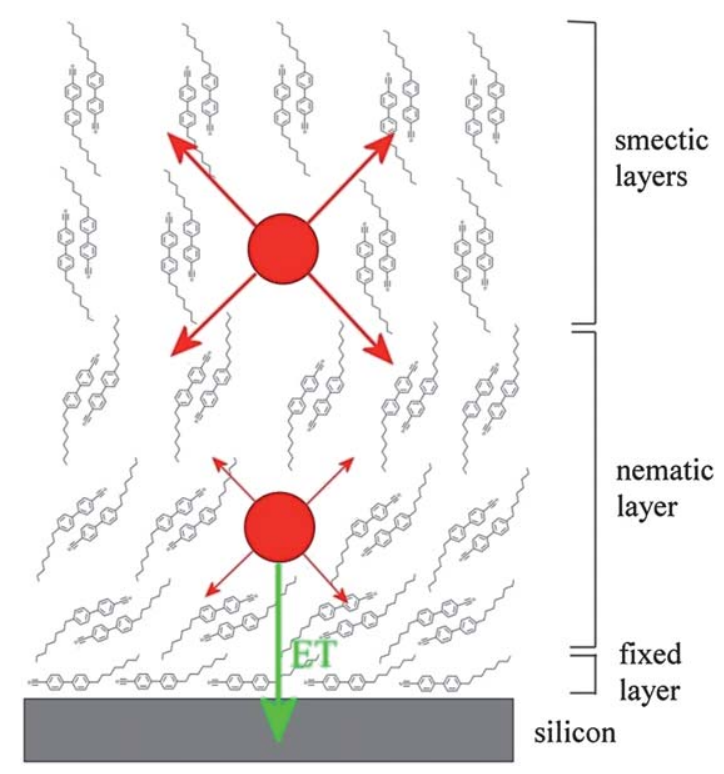

Scheme 3 LC film structure as suggested by dye diffusion measurements on no-PDI in $8 \mathrm{CB}$ on native silicon oxide after annealing.

smectic layers far away from the surface. The presence of flat smectic layers has been shown by AFM for the top $100 \mathrm{~nm}$ of LC-TF (see Fig. 2). A similar molecular structure has been proposed by Lacaze et al. ${ }^{29}$ for thin $8 \mathrm{CB}$ films on $\mathrm{MoS}_{2}$. The same authors also proved theoretically that the energy for such a transition from a smectic phase into the nematic one is comparable to the energy for layer deformation similar to the one in focal conic domains. ${ }^{30} \mathrm{~A}$ distortion of layers close to the silicon substrate was also found using scanning force polarization microscopy on wetting droplets by $\mathrm{Xu}$ et $a .^{23}$ Studies of anchoring strength and structural transitions of $8 \mathrm{CB}, 10 \mathrm{CB}$ and $12 \mathrm{CB}$ confined to alumina pores by Zumer et al. reveal a strong influence of ambient conditions on structure formation in the case of weak anchoring strength. ${ }^{31}$ Thus the observed holes in our films may occur on distortion sites due to the influence of surface induced distortions or as a memory effect of evaporated residual solvent.

\section{Comparison of LC-FCD and LC-TF structures}

With respect to structure formation the fundamentally different behavior of the $8 \mathrm{CB}$ films on native and on $100 \mathrm{~nm}$ silicon oxide are not immediately obvious. We have annealed both substrates only slightly above $\left(35{ }^{\circ} \mathrm{C}\right)$ the smectic-nematic transition $\left(33.5^{\circ} \mathrm{C}\right)$, since we found dewetting above the nematic-isotropic transition. Somewhat below the (bulk) smectic-nematic and the nematic-isotropic phase transition Garcia et al. ${ }^{4}$ observed a thick-thin (of typically 20-40 nm) coexistence, respectively, upon temperature increase, which depends on the absolute film thickness of $8 \mathrm{CB}$ on native oxide substrates. This coexistence is metastable and persists upon re-entrance into the smectic phase. However, the reason for the coexistence of different film thicknesses remains unclear. Especially the increase of the surface will cost energy. It is not obvious, why such a structure should be energetically preferable compared to a homogeneous film, as LC$\mathrm{TF}$ consists of parallel layers in the vicinity of the air-LC interface as shown in Fig. 2. Therefore the most likely explanation is that the LC-TF surface topography is a remaining instability at the smectic-nematic transition comparable with the one observed at the nematic-isotropic transition of $8 \mathrm{CB} .^{6}$ If so, the terrace formation and the observed increase in diffusion coefficient would be two independent effects as has been argued above.

$\mathrm{Xu}$ et al. ${ }^{23}$ observed steps via AFM at the edge of a smectic 8CB droplet on a $\mathrm{Si}$ wafer covered by native oxide at room temperature using scanning polarization force microscopy. They report step heights of $3.2 \mathrm{~nm}$ corresponding to an $8 \mathrm{CB}$ smectic double layer placed on a wetting trilayer (consisting of one planar monolayer and a slightly distorted smectic double layer). We suggest that our experiments can be explained by a combination of both previously reported observation: (i) a thin-thick coexistence (or "merging" $8 \mathrm{CB}$ droplets with a typical hole depth on the order of $20-40 \mathrm{~nm}$ ) and (ii) smectic steps formed at the "walls" of the holes.

Guo and Bahr ${ }^{14}$ observed the formation of FCDs in the smectic phase of thick $8 \mathrm{CB}$ droplets on native oxide upon temperature cycling well into the isotropic phase. Since we performed temperature cycling only into the nematic phase, the absence of FCDs in the current experiments is not necessarily in contradiction with those of literature data. Guo and Bahr also report $^{14}$ that $8 \mathrm{CB}$ anchoring on native oxide becomes less favored as compared to a polymer or silane coated native oxide. This observation emphasizes that the chemical and physical properties of the interface are of crucial importance for adequate wetting conditions. Moreover, long-range van der Waals interactions with the underlying $\mathrm{Si}$ substrate will have additional impact. ${ }^{32}$ Such long range interactions with Si can certainly be neglected in the case of thermally grown $(100 \mathrm{~nm})$ oxide. To the best of our knowledge, $8 \mathrm{CB}$ has not been investigated on such a substrate. For this reason we presently do not have direct experimental evidence, whether anchoring of $8 \mathrm{CB}$ on $100 \mathrm{~nm}$ oxide takes place in the same way as on native oxide. In fact previous single molecule diffusion experiments ${ }^{13}$ show differences for very thin $8 \mathrm{CB}$ films depending on the kind of substrate, which might be related to differences in the film structure close to the interface. It has to be pointed out, however, that the formation of mesoscopic structures is already observed by temperature cycling through the (bulk) smectic-nematic phase transition without entering the isotropic phase. Another reason for the different behavior of $8 \mathrm{CB}$ on the two substrates might be an increase of surface roughness due to the thermal growth process of the $100 \mathrm{~nm}$ oxide. However, our AFM experiments have shown a surface roughness of only $0.3 \mathrm{~nm}$ for both types of substrates.

Finally let us turn to the diffusion dynamics, which will be discussed separately for FCD and TF. For non-tempered $225 \mathrm{~nm}$ LC films we did not observe pronounced differences for dynamics in films prepared on native or on $100 \mathrm{~nm}$ oxide substrates. This finding is independent of whether we used single molecule tracking or FCS. After annealing (diffusion) dynamics remain the same for o-PDI on long (>20 ms) time scales (single molecule tracking), but change upon mesoscopic structure formation (FCD) on short $(<20 \mathrm{~ms})$ time scales (FCS). On the contrary, for no-PDI we observe an influence of (TF) structure formation more pronounced on long but to a much lesser extent on short time scales. To remind, o-PDI follows much more 
closely LC structures than no-PDI does. In a recent publication ${ }^{13}$ we have assigned the long time scale in analogy to bulk $8 \mathrm{CB}$ to anisotropic translational diffusion with the two components $\left(D_{\|}>D_{\perp}\right)$, while a considerable part of FCS detected dynamics on short time scales has been related to heterogeneous diffusion on short length scales.

\section{Thermally grown oxide: focal conic domain (FCD) related diffusion dynamics}

Also in the present experiments we assign the broad spread of diffusion coefficients observed on long time scales by tracking experiments on long time scales to diffusion parallel $\left(D_{\|}\right)$and perpendicular $\left(D_{\perp}\right)$ to the LC director, ${ }^{13}$ which differ in bulk $8 \mathrm{CB}$ by about a factor of 2 . For no-PDI two components $D_{1}$ and $D_{2}$ are evident from fits to the probability distribution of diffusivities on the expected time scale. Upon FCD formation the contribution of the component $D_{2}$ clearly increases. This observation can be explained by the presence of a faster or at least an increase of the faster component in the center of the FCD. Since no-PDI diffusion is not influenced by the projection of the transition dipole, the finding of faster diffusion in FCS upon FCD formation for no-PDI points to a real increase of diffusion dynamics for no-PDI.

The situation is different for o-PDI, for which we observe only one (broad) distribution of diffusion coefficients (Fig. 3). Since oPDI follows the LC director the component $D_{\|}$might be suppressed in non-structured films as the transition dipole is perpendicular to the substrate. Diffusion parallel to the director within the FCD would, however, be detected more effectively due to an increased fluorescence emission into the direction of observation (see Scheme 2). Contrary to expectations, no fast diffusion coefficient is emerging on long time scales from the broad distribution shown in Fig. 3. We therefore suggest that either $D_{\|}$and $D_{\perp}$ are of the same order of magnitude or the contribution of diffusion within the domains is too small as compared to regions outside the FCD.

Single molecule tracking results are obtained on long time scales by widefield detection, which does not directly relate diffusion to the spatial position in a FCD. This limitation is overcome by FCS which additionally extends the dynamic range to times below $20 \mathrm{~ms}$. On short time scales (FCS) we observe a slight decrease (10-20\%) of $\tau_{\mathrm{D}}$ both for o-PDI and no-PDI (see Fig. 5). This corresponds to an overall increase of decorrelation which is more pronounced for o-PDI as compared to no-PDI (see Table 3). However the finding from tracking data seems to be in contradiction to observations following FCS analysis. As already mentioned, FCS data are related also to dynamic processes other than lateral diffusion. While diffusing o-PDI molecules follow the orientation of the smectic layers within a FCD this leads to a rotation of the dielectric dipole of o-PDI which will decorrelate the fluorescence signal. The same process, however, leads to the identification of the position of a FCD, while scanning the laser focus across the sample. In the center of a FCD decorrelation becomes faster, since o-PDI follows the director orientation within a single FCD while diffusing (see Fig. 6). As it is not possible to distinguish between the two effects (translation and rotation) using FCS we cannot conclude, which one has a higher impact on our results. However both lead to a reduction of the characteristic time so that the center of the domain can be clearly identified.

The defect line in the center of the FCD is rather small, approximately on the order of one smectic layer which relates to some nanometres. ${ }^{33}$ The dimensions of the defect core are reduced with decreasing temperature as within the defect the molecules are in an undercooled isotropic phase. In the experiment the temperature is as much as $17 \mathrm{~K}$ below the isotropic phase. Given that the area of our focus is several hundred nanometres, the probability of a dye molecule being inside the defect is negligible. For this reason, the contribution of molecules entering the defect line itself is negligible within our measurements.

\section{Native oxide: terrace formation (TF) related diffusion dynamics}

For no-PDI we find a significant increase of the mean values and the spread of diffusion coefficients for LC-TF both with msd and diffusivity analysis as shown in Fig. 3 and Tables 1 and 2. o-PDI is basically not influenced by TF formation. AFM results on TF indicate that the smectic structure persists even at the walls of the holes (see Fig. 2). Since (on long time scales) there is no change of diffusion close to the walls of the holes an intriguing hypothesis is that basically diffusion parallel to the walls will be affected. Since o-PDI follows very closely the LC director we expect an increase of $D_{\|}$. However, due to the 2-D projection and in combination with the orientation of the emitting dipole such an influence is hidden both in molecule tracking and FCS experiments. Since no-PDI does not follow the orientation of the director very closely, an increase of $D_{\|}$becomes more easily detectable as is experimentally observed.

However, there might be an alternative explanation based on comparison with results from FCS. When studying LC-TF films with FCS on short time scales, one obtains the surprising result that the characteristic times $\tau_{\mathrm{D}}$ are not decreased although single molecule tracking of no-PDI does show a faster diffusion in LC-TF as compared to LC (see Fig. 3 and Tables 1 and 2). Furthermore, it is noticeable that this discrepancy only occurs for native oxide while the results on $100 \mathrm{~nm}$ oxide are consistent comparing trajectory and FCS analysis. A possible explanation might be related in the case of LC-TF to an unknown arrangement of the smectic layers close to the interface. A major difference for fluorescence measurements on native oxide as compared to thermal oxide is the presence of non-radiative transitions, ${ }^{26}$ which quench the fluorescence of molecules close to the substrate. For example, at a distance of $20 \mathrm{~nm}$ above the substrate the fluorescence intensity is reduced by more than $60 \%{ }^{26}$ This effect does not influence the measurements of single molecule tracking as the exposure time $(20 \mathrm{~ms})$ is longer than the mean first passage time. In contrast, in the case of FCS all information of areas close to the surface is suppressed in the case of both LC and LC-TF because this method only analyzes the temporal evolution of the fluorescence intensity.

Keeping this in mind we conclude, that there is no change in diffusion upon TF formation above about $40 \mathrm{~nm}$ from the surface (no changes in FCS). At the same time we observe an increase of the diffusion coefficient averaged over the total film thickness (see Fig. 3 and Table 2). A possible molecular configuration that agrees with both observations is the assumption that 
upon annealing an intermediate nematic layer (with a faster diffusion $)^{24}$ is newly formed directly above the "substrate" layer (see Scheme 3). Since we do not observe FCDs or hemicylinders, but homogeneous flat surfaces besides the terraced holes, surface domains as suggested by Zappone et al. for $8 \mathrm{CB}$ on mica $^{3}$ are probably not an explanation for the present findings for diffusion in thin $8 \mathrm{CB}$ films. Considering that the switching of the director orientation in the nematic phase can be completed within 30 $\mathrm{nm},{ }^{34}$ and assuming that the diffusion coefficient in the nematic phase is two to three times the diffusion coefficient in the smectic phase for $8 \mathrm{CB},{ }^{24}$ rough calculations would lead to an increase of the diffusion coefficient averaged over the total film of up to $30 \%$, which is in agreement with our results collected in Tables 1 and 2. The observation that this strong increase of the diffusion coefficient only occurs for the no-PDI molecules might be explained by the fact that those molecules do not fit properly in the LC matrix and thus would prefer to stay in the less oriented nematic phase. In contrast, o-PDI molecules follow the LC orientation. However the molecules are remarkably much larger than the LC molecules (see Scheme 1). For this reason o-PDI prefers the smectic phase with the higher order parameter.

\section{Conclusions}

We studied the influence of the formation of mesoscopic structures upon the diffusion of single fluorescent PDI molecules in $200 \mathrm{~nm}$ thick $8 \mathrm{CB}$ films. Depending on the thickness of the silicon oxide layer above the $\mathrm{Si}$ wafer we could identify two fundamentally different LC structures. On $100 \mathrm{~nm}$ (thermally grown) oxide we observed the formation of the well known focal conic domains (FCDs) whereas on native oxide we found a terraced structure (TF). In the latter case the analysis of the diffusion suggests that surface melting of the smectic layers occurs. In the case of the formation of FCD we could show that the two tracer molecules o-PDI and no-PDI behave differently if analyzed locally by FCS. In the case where the tracer molecule follows the LC director (o-PDI) we can map out the diffusion dynamics related directly to a single FCD. The combination of single molecule tracking and FCS allows for a detailed investigation of various aspects of mesoscopic structure formation at high spatial resolution and in combination with adapted tracer molecules. Though the presence of mesoscopic structures is evident, the physical base of TF is far from being understood. Further systematic investigations varying temperature, humidity and film thickness are needed in combination with theoretical models to systematically understand TF formation and to extend these first observations on molecular diffusion in mesoscopic liquid crystal films towards a general understanding of structure related dynamics.

\section{Acknowledgements}

We thank the German Science Foundation (DFG, FOR 877 "From Local constraints to macroscopic transport") for financial support. H. Graaf, TU Chemnitz, kindly synthesized the PDI dyes. We would like to thank one of the referees to point out to us valuable information on mesoscopic structure formation in relation to substrate properties.

\section{Notes and references}

1 J. B. Fournier, I. Dozov and G. Durand, Phys. Rev. A, 1990, 41, 2252.

2 J.-P. Michel, E. Lacaze, M. Goldmann, M. Gailhanou, M. de Boissieu and M. Alba, Phys. Rev. Lett., 2006, 96, 027803.

3 B. Zappone, E. Lacaze, H. Hayeb, M. Goldmann, N. Boudet, P. Barois and M. Alba, Soft Matter, 2011, 7, 1161.

4 R. Garcia, E. Subashi and M. Fukuto, Phys. Rev. Lett., 2008, 100, 197801.

5 D. van Effenterre, R. Ober, M. P. Valignat and A. M. Cazabat, Phys. Rev. Lett., 2001, 87, 125701.

6 S. Schlagowski, K. Jacobs and S. Herminghaus, Europhys. Lett., 2002, 57, 519.

7 M. Vilfan, T. Apih, P. J. Sebastiao, G. Lahajnar and S. Zumer, Phys. Rev. E: Stat., Nonlinear, Soft Matter Phys., 2007, 76, 051708.

8 J. J. Skaife and N. Abott, Langmuir, 2000, 16, 3529.

9 D. K. Yoon, M. C. Choi, Y. H. Kim, M. W. Kim, O. D. Lavrentovich and H.-T. Jung, Nat. Mater., 2007, 6, 866.

10 G. Friedel and F. Grandjean, Bull. Soc. Fr. Mineral. Crystallogr., 1910, 33, 409.

11 T. Kawai, S. Yoshihara, Y. Iwata, T. Fukaminato and M. Irie, ChemPhysChem, 2004, 5, 1606; T. Kawai, A. Kubota, K. Kawamura, H. Tsumatori and T. Nakashima, Thin Solid Films, 2008, 516, 2666; M. P. Lettinga and E. Grelet, Phys. Rev. Lett., 2007, 99, 197802; E. Grelet, M. Lettinga, M. Bier, R. van Roij and P. van der Schoot, J. Phys.: Condens. Matter, 2008, 20, 494213.

12 F. Kulzer, T. Xia and M. Orrit, Angew. Chem., Int. Ed., 2010, 49, 854.

13 B. Schulz, D. Täuber, F. Friedriszik, H. Graaf, J. Schuster and C. von Borczyskowski, Phys. Chem. Chem. Phys., 2010, 12, 11555.

14 W. Guo and C. Bahr, Phys. Rev. E: Stat., Nonlinear, Soft Matter Phys., 2009, 79, 011707; W. Guo, PhD thesis, Göttingen 2009.

15 F. Cichos, C. von Borczyskowski and M. Orrit, Curr. Opin. Colloid Interface Sci., 2007, 12, 272.

16 D. Täuber, M. Heidernätsch, M. Bauer, G. Radons, J. Schuster and C. von Borczyskowski, Diffus. Fundam. J., 2009, 11, 107; I. Trenkmann, D. Täuber, M. Bauer, J. Schuster, S. Bok, S. Gangopadhyay and C. von Borczyskowski, Diffus. Fundam. J., 2009, 11, 108.

17 W. Guo, S. Herminghaus and C. Bahr, Langmuir, 2008, 24, 8174.

18 I. I. Smalyukh, S. V. Shiyanovskii and O. D. Lavrentovich, Chem. Phys. Lett., 2001, 336, 88.

19 J. P. Bramble, S. D. Evans, J. R. Henderson, T. J. Atherton and N. J. Smith, Liq. Cryst., 2007, 34, 1137.

20 D. van Effenterre, R. Ober, M. P. Valignat and A. M. Cazabat, Phys. Rev. Lett., 2001, 87, 125701; D. van Effenterre and M. P. Valignat, Eur. Phys. J. E, 2003, 12, 367.

21 S. Bardon, R. Ober, M. P. Valignat, F. Vandenbrouck, A. M. Cazabat and J. Daillant, Phys. Rev. E: Stat. Phys., Plasmas, Fluids, Relat. Interdiscip. Top., 1999, 59, 6808.

22 R. M. Overney, E. Meyer, J. Frommer, H.-J. Güntherodt, G. Decher, J. Reibel and U. Sohling, Langmuir, 1993, 9, 341.

23 L. Xu, M. Salmeron and S. Bardon, Phys. Rev. Lett., 2000, 84, 1519.

24 S. V. Dvinskikh, I. Furo, H. Zimmermann and A. Maliniak, Phys. Rev. E: Stat. Phys., Plasmas, Fluids, Relat. Interdiscip. Top., 2002, 65, 061701.

25 C. Hellriegel, J. Kirstein, C. Bräuchle, V. Latour, T. Pigot, R. Olivier and S. Lacombe, J. Phys. Chem. B, 2004, 108, 14699.

26 L. Danos, R. Greef and T. Markvart, Thin Solid Films, 2008, 516, 7251.

27 S. R. Aragón and R. Pecora, J. Chem. Phys., 1976, 64, 1791.

28 A. E. Hac, H. M. Seeger, M. Fidorra and T. Heimburg, Biophys. J., $2005, \mathbf{8 8}, 317$.

29 E. Lacaze, J. P. Michel, M. Goldmann, M. Gailhanou, M. de Boissieu and M. Alba, Phys. Rev. E: Stat., Nonlinear, Soft Matter Phys., 2004, 69, 041705.

30 E. Lacaze, J.-P. Michel, M. Alba and M. Goldmann, Phys. Rev. E: Stat., Nonlinear, Soft Matter Phys., 2007, 76, 041702.

31 T. Jin, B. Zalar, A. Lebar, M. Vilfan, S. Zumer and D. Finotello, Eur. Phys. J. E, 2005, 16, 159.

32 A. Sarlah and S. Zumer, Phys. Rev. E: Stat. Phys., Plasmas, Fluids, Relat. Interdiscip. Top., 2001, 64, 051606; R. Seemann, S. Herminghaus and K. Jacobs, Phys. Rev. Lett., 2001, 86, 5534; P. Ziherl and S. Zumer, Eur. Phys. J. E, 2003, 12, 361.

33 M. Kléman, J. Phys., 1977, 38, 1511.

34 M. P. Valignat, S. Villette, J. Li, R. Barberi, R. Bartolino, E. DuboisViolette and A. M. Cazabat, Phys. Rev. Lett., 1996, 77, 1994.

35 D. Montiel, H. Cang and H. Yang, J. Phys. Chem. B, 2006, 110, 19763; M. Heidernätsch, Master thesis, TU Chemnitz, 2009. 